\title{
O Uso de Substâncias Psicoativas por Universitários Trabalhadores
}

\author{
The Use of Psychoactive Substances by Working University Students
}

\author{
Camila Teixeira Heleno', Livia de Oliveira Borges², \\ Murilo da Silva Alves ${ }^{3}$, Silvino Paulino dos Santos Neto ${ }^{4}$

\begin{abstract}
'Autora para correspondência. Universidade Federal de Minas Gerais. ORCID: 0000-0002-3203-0648. camilaheleno@gmail.com ${ }^{2}$ Universidade Federal de Minas Gerais. ORCID: 0000-0003-2251-1373. liviadeoliveira@gmail.com ${ }^{3}$ Universidade Estadual de Santa Cruz, Universidade Federal de Minas Gerais. ORCID: 0000-0001-5453-3783. murilosevla@gmail.com ${ }^{4}$ Faculdades Promove e Kennedy, Universidade Federal de Minas Gerais. ORCID: 0000-0002-6945-8736. silvinosantos.acad@gmail.com
\end{abstract}

RESUMO | Em um cenário de crescimento do uso de substâncias psicoativas entre universitários, a presente pesquisa objetivou levantar o uso destas substâncias conforme o sexo (homem e mulher), a situação de trabalho (estar trabalhando ou não) e a idade (jovem com menos de 29 anos e os demais) no contexto de duas instituições de ensino superior do setor privado com fins lucrativos em Belo Horizonte. Do ponto de vista metodológico, aplicamos questionários estruturados em 868 estudantes em sessões coletivas em sala de aula. Todos os participantes responderam anonimamente. Desenvolvemos análises estatísticas das respostas. A análise fatorial identificou dois tipos de sustâncias psicoativas - Prescritas e Não Prescritas. As análises de regressão mostraram que apenas o sexo apresenta capacidade preditiva dos escores nos fatores. $O$ uso de café como substância estimulante é mais frequente entre os estudantes trabalhadores e com mais de 29 anos. Os resultados podem ser importantes para a construção de instrumento específico, bem como para orientar novos levantamentos acerca da dinâmica do trabalho influenciando tais condutas.

PALAVRAS-CHAVE: Estudantes universitários. Trabalho. Abuso de substâncias psicoativas. Validade.

\begin{abstract}
Given the growth in the use of psychoactive substances among university students, the present research aimed to investigate the use of these substances by sex (men and women), situation of work (to be work or no), and age (younger than 29 years old and older). The research was carried out in two institutions in the private sector in Belo Horizonte. Regarding the methodology, structured questionnaires were applied to 868 students, which were answered anonymously. The responses were analyzed statistically. The factorial analysis identified two types of psychoactive substances - Prescribe and Nonprescribed ones. Regression analysis showed that only sex presents predicting capacity to scores in the factors. The use of coffee as a stimulating substance is more frequent among students that are workers and who are older than 29 years. The results can be important for the design of a specific instrument, as well as to direct new research about the dynamics of work influencing such behaviors.
\end{abstract}

KEYWORDS: College students. Work. Psychoactive substances abuse. Validity. 


\section{O uso de substâncias psicoativas por universitários trabalhadores}

As substâncias psicoativas (SPAs) alteram o comportamento, a consciência, o humor e a cognição, agindo no sistema nervoso central (Edwards, Arif, \& Hadgson, 1981). São consideradas SPAs drogas lícitas ou ilícitas, podendo ser classificadas de acordo com as suas modificações observáveis na atividade mental em depressoras (álcool, barbitúricos, benzodiazepínicos, opioides, solventes ou inalantes), estimulantes (anfetaminas, cocaína), perturbadoras (maconha, ecstasy, ácido lisérgico -LSD) e outras drogas, que não estão em uma única categoria (tabaco, cafeína, esteroides anabolizantes) (Nicastri et al., 2008). O aumento da prevalência e dos riscos decorrentes do uso de substâncias psicoativas (SPAs) por universitários nas últimas décadas tem sido apontado em diversos estudos como motivo de preocupação de pesquisadores, sociedade e Estado (Andrade, Duarte, \& Oliveira, 2010; Chiapetti \& Serbena, 2007; Eckschmidt, Andrade, \& Oliveira, 2013; Lemos et al., 2007; Pelicioli, Barelli, Gonçalves, Hahn, \& Scherer, 2017; Silva \& Tucci, 2015).

No Brasil, as pesquisas (p. ex., Lemos et al., 2007; Chiapetti \& Serbena, 2007; Silva \& Tucci, 2015; Andrade et al., 2010) apontaram os universitários como população potencialmente vulnerável à iniciação, manutenção e aumento do uso de SPAs por diversos motivos, como: exigências, demandas e condições da formação/trabalho, permissividade social, autonomia, poder aquisitivo, acesso a SPAs, liberdade, experimentação, formação grupal, situações de estresse e recreativas. Neste sentido, o "I Levantamento Nacional sobre o Uso de Álcool, Tabaco e Outras Drogas entre Universitários das 27 Capitais Brasileiras" (Andrade et al., 2010) apontou que o consumo de álcool, tabaco e outras drogas é mais frequente entre os estudantes universitários do que na população em geral, e que $48,7 \%$ dos universitários pesquisados já experimentaram alguma droga ilícita pelo menos uma vez na vida. Segundo Andrade et al. (2010), houve crescimento significativo na prevalência do uso por universitários, entre 1996 e 2001, das seguintes SPAs: álcool (de 88,5 para $91,9 \%$ ), tabaco (de 42,8 para $50,5 \%$ ), maconha (de 31,3 para $35,3 \%$ ), inalantes (de 17,9 para $24,5 \%$ ), alucinógenos (de 6,1 para $11,4 \%$ ), anfeta- mínicos (de 4,8 para 9,0\%), anticolinérgicos (de 1,1 para $2,9 \%$ ) e barbitúricos (de 1,0 para $1,7 \%$ ).

O uso de SPAs é um fenômeno complexo e multidimensional, que não pode ser explicado por causas únicas. Pelo contrário, são reconhecidas a influência e interação dos fatores biológicos, psicológicos, sociais, econômicos, políticos e contextuais (familiares, escolares e grupais), assim como, valores, crenças e trabalho (Cogollo-Milanés et al., 2011; Martins \& Zeitoune, 2007). Apesar da ligação com outras variáveis de influência no uso, apontadas nos estudos - como pares, família, etnia/raça/cor, gênero, condições sociais/econômicas, desenvolvimento psicológico e social do indivíduo, mídia, comunidade, suporte social e religião - não é possível generalizar sobre a capacidade preditiva e protetiva destas variáveis para o uso de SPAs (Chiapetti \& Serbena, 2007; Andrade et al., 2010; Oliveira, Cunningham, Strike, Brands, \& Wright, 2009).

De modo geral, o caráter deletério do uso/dependência de SPAs evidencia-se pelos impactos sociais e econômicos gerados, caracterizando-se também como um problema de saúde pública. Apesar dos impactos citados, existe uma manutenção controversa da lógica do capital que sustenta as causas geradoras, no trabalho e nas universidades, sendo - uso das SPAs uma solução eficaz para suportar as exigências impostas por estes locais (Eckschmidt et al., 2013; Lima, 2010; Pereira, Souza, Buaiz, \& Siqueira, 2008).

O uso de SPAs adquire a nomenclatura de uso "funcional" quando permite aumentar o desempenho e suportar as atividades laborais, e "disfuncionais" quando se tornam um círculo vicioso, sendo assim, problema e solução no trabalho (Lemos et al., 2007; Lima, 2010). Neste sentido, ser estudante e trabalhador, expõe o universitário ao acúmulo de exigências e situações impostas pelas tarefas e condições geradoras de estresse, ligadas tanto à atividade de trabalho, quanto às vivenciadas no ambiente de formação acadêmica, estabelecendo uma condição vulnerável ao consumo de SPAs lícitas e ilícitas, e às suas consequências (Andrade et al., 2010; Oliveira et al., 2009; Padovani et al., 2014). 
Além disso, o uso de SPAs entre universitários trabalhadores aumenta a probabilidade de morbidade e mortalidade, por propiciar e expor a outros comportamentos de risco à saúde, e também a consequências, como: violência interpessoal, homicídios, suicídio, comportamento sexual de risco, uso inconsistente de preservativos, aumento da incidência de doenças infectocontagiosas, acidentes com veículos automotores, incapacidades pós-trauma, distúrbios do sono, experiências psicóticas, mudanças do hábito alimentar, prejuízo do desempenho atlético, mortes prematuras, redução em anos de vida potencialmente e/ou a diminuição da expectativa de vida dos universitários, entre outros efeitos (Andrade et al., 2010; Cogollo-Milanés et al., 2011; Fonseca-Pedrero, Ortuño-Sierra, Paino, \& Muñiz, 2016; Lima et al., 2017; Pelicioli et al., 2017).

As consequências e a dependência de SPAs podem levar a prejuízos acadêmicos, como perda do rendimento, falta de atenção durante as aulas, ausências, atraso e maior sonolência, podendo acarretar também problemas no trabalho, punições, absenteísmo, rebaixamento e isolamento do trabalhador (Eckschmidt et al., 2013; Lima, 2010; Lima et al., 2017; Pereira et al., 2008).

No contexto dos universitários trabalhadores, 0 incentivo governamental e o sistema de crédito estudantil, bolsas e financiamento do ensino superior no Brasil, foram decisivos para as instituições privadas atenderem $\circ$ público trabalhador, ampliando de maneira significativa a oferta de cursos noturnos. No entanto, poucas são as pesquisas sobre o tema em tela com universitários no sistema privado de ensino, principalmente quando se agregam as características de ser universitário e trabalhador. Os universitários trabalhadores são considerados um grupo de investimento social do país, mas, dentro do panorama de vulnerabilidades, unem duas atividades (estudo e trabalho) que predispõem a situações de estresse e a influências nocivas sobre sua saúde mental (Padovani et al., 2014).

De todo modo, existem indícios de uma dependência mimética desenvolvida entre o uso de SPAs e as exigências e demandas do trabalho, universidade e vida, considerada como uma ferramenta de integração e potencializadora de suporte e interação social. A falta de desenvolvimento da socialização saudável, devido ao ambiente estressante da academia e do trabalho contribui para o uso de SPAs (Díaz \& Gómez, 2007).

Neste contexto, este estudo foi planejado com o objetivo de investigar o uso de substâncias psicoativas entre universitários trabalhadores de duas faculdades privadas de Belo Horizonte/MG. Para tanto, analisaremos a frequência do uso de substâncias psicoativas segundo a situação de trabalho (trabaIha e não trabalha), a idade (ser jovem ou adulto) e o sexo (masculino ou feminino) do estudante universitário. Para efeito de delimitação, são considerados jovens as pessoas que têm entre 15 a 29 anos de idade, de acordo com o Estatuto da Juventude (Lei no 12.852 , de $05 / 08 / 2013$ ).

Tendo em vista a consecução de tal objetivo, elaboramos também questões de pesquisa mais específicas, visando orientar o desenvolvimento da coleta e análise dos resultados, a saber:

1) As substâncias psicoativas podem ser agrupadas a partir do uso que os universitários dizem realizar?

2) Quanto os universitários da amostra afirmam usar substâncias psicoativas?

3) O uso de substâncias psicoativas varia conforme a situação de trabalho, a idade e o sexo dos universitários?

\section{Método}

Realizamos um survey com estudantes universitários de duas faculdades privadas com fins lucrativos da cidade de Belo Horizonte/MG.

\section{Participantes}

Participaram desta pesquisa, respondendo de modo válido, um total de 868 estudantes universitários. Os estudantes cursavam diferentes cursos superiores (bacharelados e tecnólogos) em três unidades localizadas em diferentes regiões da cidade (Oeste, Centro-Sul e Venda Nova). Apresentamos a caracterização dos participantes na Tabela 1. 
Tabela 1. Caracterização dos estudantes universitários considerando idade, sexo e situação de trabalho

\begin{tabular}{|c|c|c|c|c|c|c|c|}
\hline & \multirow{2}{*}{ Idade } & & \multirow{2}{*}{\multicolumn{2}{|c|}{ Sexo feminino* }} & \multicolumn{3}{|c|}{ Situação de trabalho } \\
\hline & & & & & Trabalha & Não trabalha & \\
\hline Média & DP & $\mathrm{N}$ & $f(\% *)$ & $\mathrm{N}$ & $f(\% *)$ & $f(\% *)$ & $\mathrm{N}$ \\
\hline 28,11 & 9,56 & 831 & $\begin{array}{c}478 \\
(57,0 \%)\end{array}$ & 838 & $598(74,3 \%)$ & $207(25,7 \%)$ & 805 \\
\hline
\end{tabular}

*Percentual válido.

\section{Instrumentos}

Os participantes foram solicitados a responder: a) uma ficha com questões solicitando informações para descrever os participantes em relação ao sexo (masculino=1 e feminino=2) situação de trabalho (não trabalha $=1$ e trabalha=2) e à idade (variável contínua); e, b) um questionário estruturado, que solicita informações sobre o uso atual de diferentes substâncias psicoativas (álcool, cigarro, café, maconha, cocaína, ecstasy, antidepressivos, estabilizadores de humor/antipsicóticos e ansiolíticos/hipnóti$\cos$ ), indicando a sua frequência de uso (nunca $=1$, mensalmente $=2$, semanalmente $=3$ ou diariamente $=4)$.

As questões fazem parte de um protocolo de pesquisa internacional mais amplo (Workforall Consortium, 2014), que tem sido aplicado em diferentes países (p. ex., Espanha e México) e foram traduzidas para aplicação no Brasil. Não encontramos, entretanto, literatura que abordasse as evidências de validade e consistência do referido questionário.

\section{Procedimentos de coleta de dados}

A coleta de dados foi realizada de forma coletiva por uma equipe de estudantes de graduação e pós-graduação em psicologia, previamente treinada, sob a autorização das instituições de ensino e com apoio dos professores universitários. Após a assinatura do Termo de Consentimento Livre e Esclarecido (TCLE) aplicamos os questionários em formato tradicional (papel e lápis) nas salas de aula. O anonimato dos participantes foi preservado pela não identificação destes nos questionários e armazenamento em local diferente dos TCLEs. A pesquisa foi aprovada pelo Comitê de Ética em Pesquisa sob a designação: CAAE 37058714.7.0000.5149

\section{Procedimentos de análise dos dados}

As respostas dos participantes foram registradas em um banco de dados no SPSS for Windows para realização de análises estatísticas descritivas, análise fatorial do questionário de uso de substâncias psicoativas, estimativas dos coeficientes de consistência por fator (coeficiente alfa de Cronbach) e análises de regressão linear múltipla (stepwise), análise de variância e correlação.

As estatísticas descritivas foram necessárias para descrever a amostra, como avaliação preliminar para as demais análises e para responder a segunda questão de pesquisa. A análise fatorial e estimativa do alfa de Cronbach permitiram desenvolver análises com escores nos fatores com confiabilidade empiricamente conhecida, além de necessária para responder a primeira e segunda questões de pesquisa. As três últimas análises citadas (análises de regressão linear múltipla stepwise), análise de variância e correlação) exploram a variância do uso de substâncias psicoativas pelas três variáveis antecedentes (sexo, situação de trabalho e idade). $O$ detalhamento da aplicação das técnicas será exposto na proporção que expomos os resultados.

\section{Resultados}

Apresentamos os resultados descritivos considerando a quantidade de estudantes universitários que relataram fazer uso de diferentes substâncias psicoativas na Tabela 2. Nessa Tabela, observamos que a quase totalidade das distribuições de frequência tende à concentração das respostas no nível mais baixo da escala (Nunca), o que consideramos aceitável em se tratando de uso de substâncias psicoativas. Excetuou-se à tal tendência a distribuição de 
frequência relativa ao uso do café, que além de tendência inversa traça uma curva quase parabólica, ou em formato de $U$, com grande concentração no nível mais baixo da escala e uma tendência crescente em relação a outros níveis. Diferente também (embora menos) está a distribuição dos escores referentes ao uso de cocaína com a maior concentração no nível "Nunca" da escala. Em outras palavras, as respostas acerca do uso da cocaína têm um mínimo de variância sendo quase uma constante e não uma variável propriamente dita.

Tabela 2. Resultados descritivos do uso de substâncias psicoativas entre estudantes universitários

\begin{tabular}{lccccc}
\hline \multicolumn{1}{c}{ Substância psicoativa } & $\mathrm{N}$ & $\begin{array}{c}\text { Nunca } \\
\mathrm{f}(\% *)\end{array}$ & $\begin{array}{c}\text { Mensal } \\
f(\% *)\end{array}$ & $\begin{array}{c}\text { Semanal } \\
f(\% *)\end{array}$ & $\begin{array}{c}\text { Diário } \\
f(\% *)\end{array}$ \\
\hline Café & 851 & $145(17,0 \%)$ & $61(7,2 \%)$ & $112(13,2 \%)$ & $533(62,6 \%)$ \\
Álcool & 842 & $366(43,5 \%)$ & $193(22,9 \%)$ & $271(32,2 \%)$ & $12(1,4 \%)$ \\
Tabaco & 827 & $715(86,5 \%)$ & $28(3,4 \%)$ & $24(2,9 \%)$ & $60(7,3 \%)$ \\
Ansiolíticos/hipnóticos & 836 & $751(89,8 \%)$ & $39(4,7 \%)$ & $20(2,4 \%)$ & $26(3,1 \%)$ \\
Maconha & 838 & $787(93,9 \%)$ & $26(3,1 \%)$ & $19(2,3 \%)$ & $6(0,7 \%)$ \\
Antidepressivos & 836 & $793(94,9 \%)$ & $14(1,7 \%)$ & $10(1,2 \%)$ & $19(2,3 \%)$ \\
Estabilizadores de & 834 & $808(96,9 \%)$ & $12(1,4 \%)$ & $2(0,2 \%)$ & $12(1,4 \%)$ \\
humor/antipsicóticos & 831 & $818(98,4 \%)$ & $11(1,3 \%)$ & $1(0,1 \%)$ & $1(0,1 \%)$ \\
Ecstasy & 837 & $831(99,3 \%)$ & $4(0,5 \%)$ & -- & $2(0,2 \%)$ \\
Cocaína & & & & & \\
\hline
\end{tabular}

*Percentual válido

Em um segundo momento, selecionamos aleatoriamente 300 participantes do total, pela rotina do SPSS, com intuito de explorar a estrutura interna do instrumento sobre SPAs, porque a amostra total é mais extensa do que o desejável para aplicar tal análise. Realizamos preliminarmente análises de componentes principais. Na primeira aplicação da técnica, verificamos que os itens referentes ao uso do café e maconha apresentaram communalities respectivamente de 0,03 e 0,27. O primeiro também não apresentou nenhum coeficiente de correlação superior a 0,30 , corroborando o que já havíamos assinalado na Tabela 2. Isto significa que ele não faz parte do mesmo conjunto de itens dos demais, ou seja, não é indicador do mesmo fenômeno. $O$ segundo item citado tinha uma correlação de 0,36 com o referente ao uso de ecstasy. Por isso, voltamos a aplicar a técnica excluindo o item referente ao café.

Para verificar a adequação dos dados à análise, consideramos: a medida de Kaiser-Meyer-Olkin of Sampling Adequacy (KMO), cujo resultado foi de 0,63; o teste de esfericidade de Bartlett $(\chi 2=356.905 \mathrm{e}$ $\mathrm{p}<0,001$ ); a variação das communalities de 0,27 a 0,69 , sendo que apenas o item referente ao uso de maconha era inferior a 0,30 ; e o fato de todos os itens apresentarem coeficientes de magnitude superior a 0,30 e nenhum ultrapassava 0,70 na matriz de correlação. $O$ coeficiente $K M O$ não corresponde exatamente ao desejável. Entretanto, consideramos que questões sobre $\circ$ uso de SPAs são delicadas e nossa amostra bastante diversificada em decorrência das características próprias das instituições de ensino em que desenvolvemos a pesquisa. Além disso, tal questionário estruturado não dispunha deste tipo de análise anteriormente. Decidimos, então, pela continuidade da análise mesmo em caráter exploratório, na certeza de gerar contribuições para o desenvolvimento do instrumento.

A decisão sobre o número de fatores foi realizada após a observação do screeplot, que apontou estrutura com dois componentes. Aplicamos, então, a análise fatorial (Principal axis factoring) com rotação oblíqua, mas a correlação entre os fatores era de apenas $-0,05$. Por isso, optamos pela mesma análise fatorial com rotação varimax. Apresentamos, então, os resultados com as cargas fatoriais, superiores a 0,30, na Tabela 3. 
Tabela 3. Cargas fatoriais (superiores a 0,30 ) extraídas pela análise fatorial (rotação varimax)

\begin{tabular}{lcc}
\hline \multicolumn{1}{c}{ Fatores } & $\begin{array}{c}\text { Substâncias } \\
\text { Prescritas }\end{array}$ & $\begin{array}{c}\text { Substâncias } \\
\text { Não Prescritas }\end{array}$ \\
\hline Eigenvalues (valor critério) & 2,03 & 1,89 \\
\hline Proporção de explicação da variância & 25,40 & 23,60 \\
\hline Coeficientes Alfa de Cronbach & 0,71 & 0,46 \\
\hline Antidepressivos & 0,82 & \\
Estabilizadores de humor/antipsicóticos & 0,68 & \\
Ansiolíticos/hipnóticos & 0,59 & 0,60 \\
Álcool & & 0,54 \\
Maconha & & 0,51 \\
Tabaco & & 0,47 \\
Ecstasy & & --- \\
Cocaína & --- & \\
\hline
\end{tabular}

O total da variância explicada foi de $49 \%$. Os fatores foram nomeados como Substâncias Prescritas e Substâncias Não Prescritas, sendo importante observar que o segundo reúne substâncias aceitas socialmente e ilícitas em oposição àquelas que necessitam (ou deveriam necessitar) ser receitadas por um profissional que compõe o primeiro fator. $O$ item referente ao uso de cocaína não carregou em nenhum dos fatores, refletindo as características de sua distribuição vista nas estatísticas descritivas.

Para a amostra completa da pesquisa, calculamos então os escores para cada fator pela média ponderada dos seus itens pelas cargas fatoriais, tendo em vista refletir sobre as diferenças de contribuição de cada item para a formação do fator (Tabela 4).

Tabela 4. Escores nos fatores da escala de uso de substâncias psicoativas

\begin{tabular}{lccccccc}
\hline \multicolumn{1}{c}{ Fatores } & & \multicolumn{3}{c}{ Desvio- } \\
& N & Mínimo & Máximo & Média & padrão & Variância \\
\hline Substâncias Prescritas & 844 & 0,39 & 4,00 & 1,10 & 0,40 & 0,16 \\
Substâncias Não Prescritas & 853 & 0,24 & 3,27 & 1,34 & 0,43 & 0,18 \\
\hline
\end{tabular}

A partir disso, realizamos duas análises de regressão linear múltipla (stepwise), uma para cada fator (Substâncias Prescritas, Substâncias Não Prescritas) como variável critério (ou variáveis tomadas como "y" na análise de regressão) e sexo (masculino e feminino), situação de trabalho (não trabalha e trabalha) e idade (variável contínua) como variáveis antecedentes, ou tomadas como " $x$ " na análise de regressão (Tabela 5). 


\begin{tabular}{|c|c|c|c|c|c|}
\hline \multirow[b]{2}{*}{ Modelos } & \multicolumn{2}{|c|}{$\begin{array}{c}\text { Coeficientes Não } \\
\text { Padronizados }\end{array}$} & \multirow{2}{*}{$\begin{array}{c}\text { Coeficientes } \\
\text { Padronizados } \\
\text { Beta } \\
\end{array}$} & \multirow[b]{2}{*}{$t$} & \multirow[b]{2}{*}{ Sig. } \\
\hline & B & Erro Padrão & & & \\
\hline \multicolumn{6}{|c|}{ Variável Critério: Fator 1 - Substâncias Prescritas $\left(R^{2}\right.$ ajustado $=0,01, F=7,47$ para $\left.p=0,006\right)$} \\
\hline (Constant) & 0,99 & 0,05 & & 19,53 & $<0,001$ \\
\hline Sexo & 0,08 & 0,03 & 0,10 & 2,73 & 0,006 \\
\hline \multicolumn{6}{|c|}{ Variável Critério: Fator $2-$ Substâncias Não Prescritas $\left(R^{2}\right.$ ajustado $=0,03, F=20,30$ para $\left.p<0,001\right)$} \\
\hline (Constant) & 1,56 & 0,05 & & 30,93 & $<0,001$ \\
\hline Sexo & $-0,14$ & 0,03 & $-0,16$ & $-4,53$ & $<0,001$ \\
\hline
\end{tabular}

Ambas as análises de regressão múltipla, apesar da opção pela técnica stepwise, produziram apenas um modelo para cada fator de uso de SPAs pelo fato de exclusivamente a variável antecedente sexo oferecer alguma predição aos escores nos fatores em equações com um poder de explicação muito baixo. No uso das Substâncias Prescritas as mulheres apresentaram média $(M=1,14 ; d p=0,46)$ superior ao dos homens $(M=1,06 ; d p=0.32)$. Em relação às Substâncias Não Prescritas, a tendência se inverte, de modo que a média masculina $(M=1,42 ; d p=0,45)$ é maior que a feminina $(M=1,28 ; d p=0,40)$.

Acerca do uso de café, devido sua distribuição distinta dos demais itens, tomamos este como variá- vel critério e aplicamos análise variância adotando sexo, situação de trabalho e idade (ter ou não mais que 29 anos) como variáveis antecedentes (Tabela 6). Os resultados indicaram haver variância conjunta significativa do uso desta substância pelas duas últimas variáveis antecedentes, sendo que: os universitários que trabalham tendem a usá-la mais $(M=3,33 ; d p=1,10)$ do que aqueles que não trabaIham $(M=2,90 ; d p=1,25)$; e os jovens usam menos $(M=3,08 ; d p=1,20)$ do que os demais $(M=3,47$; $d p=1,03)$. O coeficiente Eta indica, entretanto, baixo poder explicativo. Por fim, estimamos a correlação (Sperman's rho) entre o uso do café e a idade (como variável contínua) e encontramos coeficiente não significativo $(r=0,04 ; p=0,41)$.

Tabela 6. Análise de variância do uso do café por sexo, faixa de idade e situação de trabalho

\begin{tabular}{|c|c|c|c|c|}
\hline Fonte & $\mathrm{F}$ & Sig. & $\begin{array}{l}\text { Eta Parcial } \\
\text { (quadrado) }\end{array}$ & Poder observado ${ }^{a}$ \\
\hline Modelo corrigido & 6,24 & $<0,001$ & 0,06 & 1,00 \\
\hline Intercepto & 3276,37 & $<0,001$ & 0,82 & 1,00 \\
\hline Sexo & 2,08 & 0,15 & 0,01 & 0,30 \\
\hline Situação de trabalho & 14,09 & $<0,001$ & 0,02 & 0,31 \\
\hline $\begin{array}{l}\text { Idade (menos e mais que } \\
29 \text { anos) }\end{array}$ & 7,70 & 0,006 & 0,01 & 0,79 \\
\hline $\begin{array}{l}\text { Situação de trabalho * } \\
\text { Sexo }\end{array}$ & 0,36 & 0,547 & $<0,01$ & 0,09 \\
\hline Sexo* Idade & 0,53 & 0,47 & 0,001 & 0,11 \\
\hline $\begin{array}{l}\text { Situação de trabalho * } \\
\text { idade }\end{array}$ & 0,07 & 0,80 & $<0,01$ & 0,06 \\
\hline $\begin{array}{l}\text { Situação de trabalho * } \\
\text { idade*sexo }\end{array}$ & $<0,01$ & 0,96 & $<0,01$ & 0,05 \\
\hline
\end{tabular}




\section{Discussão}

Em relação à primeira questão de pesquisa - As substâncias psicoativas podem ser agrupadas a partir do uso que universitário dizem realizar? -, a análise fatorial mostrou que é possível agrupar empiricamente as substâncias psicoativas, porém a escala usada na pesquisa demanda melhorias. Os fatores identificados são teoricamente pertinentes (além da base empírica), mas é preciso repensar - modelo de questão para tornar possível contar com um aumento da confiabilidade do questionário. É pertinente também considerar a inclusão de questões além das relacionadas ao uso pessoal, como a observação do uso pelos colegas.

O fato da rotação varimax (ortogonal) ter sido mais adequada que a oblíqua é um achado importante: os participantes distinguem sem sobreposições o uso de SPAs prescritas das demais. Em outras palavras, a prescrição médica de uma SPA é um distintivo forte do uso delas.

O uso do café como não fazendo parte do atual conjunto de itens é outro resultado importante. Apesar de ser uma SPA, não se agrupa com as demais. Seu uso é socialmente muito aceito e para agrupá-lo com outros itens demandaria introduzir outros de igual aceitação na cultura brasileira.

É provável que o uso da cocaína tenha se apresentado próximo a uma constante pela possível contaminação da resposta pela desejabilidade social, - que fortalece a sugestão já apresentada de repensar a formulação da questão. Entretanto, não devemos descartar a possibilidade deste resultado refletir uma característica específica da amostra como haver outras drogas ilícitas mais típicas deste segmento populacional.

Acerca da segunda questão de pesquisa - Quanto os universitários da amostra afirmam usar substâncias psicoativas? - há mais de uma consideração a tecermos aqui. A primeira diz respeito a tendência marcante de negar o uso de SPAs na maioria dos itens e fatores encontrados, reforçando a interpretação dos resultados como contaminados pela desejabilidade social. Mas no item acerca do uso do álcool, apesar de no geral tal tendência ser manti- da, o número de pessoas respondendo com os níveis da escala "mensal" e "semanal" é diferenciado dos demais itens. Esse aspecto deve ser considerado em um replanejamento da forma de perguntar. A distribuição parabólica das frequências no item sobre o café pode ser compreendida como uma distribuição bimodal que valoriza os extremos (nunca usar ou usar diariamente), embora o uso diário seja cerca de 3,5 vezes mais que o outro extremo. Essas peculiaridades devem nos conduzir a indagar sobre a adequação dos níveis da escala. Os participantes não tiveram como indicar usos que estão, por exemplo, entre o mensal e o semanal, entre o semanal e o diário, que são frequentes no dia e/ou que são irregulares na frequência.

Em relação à terceira questão de pesquisa - $O$ uso de SPAs varia conforme a situação de trabalho, a idade e o sexo dos universitários? - precisamos tecer nossas considerações aqui passo a passo. O primeiro achado em direção desta pergunta é que das três variáveis antecedentes abrangidas na pesquisa (idade, sexo e situação de trabalho) apenas sexo ofereceu predição aos escores dos dois fatores (Substâncias Prescritas e Substâncias Não Prescritas).

Ser mulher aumenta a probabilidade de uso de substâncias prescritas. Esse resultado está em conformidade com a literatura em geral que indica que as estudantes universitárias são mais adeptas e predispostas ao seu uso que os estudantes (Fiorini et al., 2003; Lemos et al., 2007). Ainda assim, existem estudos (p. ex., Teixeira, Souza, Buaiz, \& Siqueira, 2010) que encontraram, por exemplo, um consumo de anfetamínicos e ansiolíticos semelhante entre os sexos. Entre as substâncias mais utilizadas por elas foram identificadas na literatura 0 uso de medicamentos psicotrópicos, bem como tranquilizantes, barbitúricos, ansiolíticos, alucinógenos e medicamentos para emagrecer (Andrade et al., 2010; Fiorini et al., 2003; Lemos et al., 2007; Mardegan, Souza, Buaiz e Siqueira, 2007; Pereira et al., 2008).

A literatura indica algumas causas para este maior uso de substâncias prescritas na população feminina. Padovani et al. (2014), por exemplo, sinalizaram que a existência de elevadas taxas de prevalência para sintomas de ansiedade e depressão em 
estudantes universitários, comparadas à população geral, aponta para uma maior medicalização por parte delas. Segundo os autores, os sintomas de burnout e estresse também estavam mais presentes entre elas, alertando para sua maior vulnerabilidade. Segundo Picolotto, Libardoni, Migott e Geib (2010), as substâncias surgem como meio de alívio das adversidades psíquicas, sem atuar nas causas. De todo modo, dadas as implicações negativas do sofrimento psicológico para o processo de aprendizagem, formação do futuro profissional (Padovani et al., 2014) e para a vida dos estudantes, é preciso que as instituições de ensino, famílias e sociedade estejam atentas a estas questões.

No entanto, também identificamos que, para o uso de substâncias prescritas, a idade do estudante e $\circ$ fato de estar (ou não) trabalhando não interferem em seu consumo. Este último resultado sinaliza que o trabalho destes estudantes pode não ser adoecedor ou, mais provavelmente, que o fato de estudar é percebido como condição mais penosa e/ou que a heterogeneidade de trabalhos da amostra mascarou possíveis efeitos. Pesquisas futuras, aprofundando na natureza e nas condições do trabalho exercido pelos universitários serviriam para esclarecer esta questão.

Retomando a variação do sexo no uso de SPAs considerando o sexo do estudante universitário, identificamos que ser homem aumenta a probabilidade de uso de Substâncias Não Prescritas. O maior uso de substâncias socialmente aceitas por estudantes universitários do sexo masculino também já havia sido identificado na literatura. Lima et al. (2017), Fiorini et al. (2003) e Mardegan et al. (2007), por exemplo, também encontraram que os estudantes do sexo masculino são mais adeptos do uso de substâncias não prescritas, incluindo bebidas alcoólicas e drogas ilícitas. Também já fora encontrado um maior uso entre eles de tabaco (Lima et al., 2017; Pereira et al., 2008; Teixeira et al., 2010), maconha (Andrade et al., 2010; Pereira et al., 2008; Teixeira et al., 2010), ecstasy (Andrade et al., 2010) e principalmente de álcool (Andrade et al., 2010; Lemos et al., 2007; Lima et al., 2017).

Em relação ao consumo de tabaco, em especial, diferentemente do encontrado nesta pesquisa (conjuntamente com outras SPAs) a diferença entre se- xos não foi percebida por Andrade et al. (2010). Lima et al. (2017), haviam encontrado que menos de $2 \%$ dos estudantes de sua amostra declararam fumar diariamente pelo menos um cigarro. Na presente pesquisa este resultado foi superior $(7,3 \%)$ ao de Lima et al. (2017) mas inferior $(25,7 \%)$ ao de Pereira et al. (2008) reforçando a noção, apresentada pelos primeiros autores, de que divergências entre prevalências podem ocorrer devido às características sociodemográficas e culturais específicas de cada população, mais que pelo sucesso das campanhas e/ou pelas ações de combate ao tabagismo no Brasil.

Especificamente em relação ao consumo de álcool, os resultados reforçam a noção de que este é uma das drogas mais consumidas pelos universitários (Lima et al., 2017), em estudo que não considerou o uso de café, com 56,5\% dos estudantes relatando algum uso. Seu uso maior pelos universitários do sexo masculino corroboram a literatura da área (Andrade et al., 2010; Lemos et al., 2007; Lima et al., 2017) embora alguns artigos não tenham encontrado tal relação (p. ex., Teixeira et al., 2010).

A literatura também indica algumas explicações para o maior uso de álcool entre os estudantes do sexo masculino. Por exemplo, segundo Lima et al. (2017), os estudantes do sexo masculino, de modo geral, são mais propensos a comportamentos de risco. Segundo os autores, a explicação destes comportamentos está relacionada à influência social e a fatores culturais de demonstração de poder. E segundo Andrade et al. (2010), os homens usam mais maconha, inalantes, cloridrato de cocaína, alucinógenos, ecstasy e esteroides anabolizantes que as mulheres, para todas as medidas de uso. Partindo de outros autores (p. ex., Dázio, Zago, \& Fava, 2016) poderíamos levantar que o maior uso pelos universitários homens deste tipo de SPAs podem ser uma expressão de sua construção de gênero, em que se reproduz as práticas hegemônicas socialmente tomadas como típicas do gênero masculino.

Outro passo de nossas considerações motivadas pela terceira questão focaliza o uso do café como SPA estimulante embora analisado separadamente com base em item isolado. Trabalhar e o avanço da idade aumentam a probabilidade de uso dessa substância estimulante. 
Esta relação com a situação de trabalho é apontada na literatura, mostrando que por passar boa parte da vida no meio laboral, os ritmos e as complexidades que o trabalho alcançou neste mundo moderno por si só constituem um alto risco quanto ao uso de substâncias psicoativas (Fontaine, 2006; Martins \& Zeitoune, 2007). Fontaine (2006), Martins e Zeitoune, (2007), Lima (2010) e Pelicioli et al. (2017), por exemplo, identificaram como propulsores do uso de SPAs a ansiedade, angústia, insatisfação e estresse desencadeado pelo trabalho, devido: condições e ambiente de trabalho, exposições de cargas (físicas e mentais), exigências produtivas (desempenho, competências), conteúdo do trabalho, tensões da profissão, organização institucional e do trabalho, relações interpessoais e socialização, tendo estas variáveis, impactos diferentes de acordo com os contextos específicos a que o trabalhador está submetido. Existindo fatores de risco tanto quantitativos (produtividade, pressões de tempo), como qualitativos (trabalhos com pouca criatividade e interação social) que podem potencializam o uso de drogas nos trabalhadores.

São uma concordância para Fontaine (2006) e Lima (2010) que o consumo de SPAs é considerado "lubrificante", "suporte" ou "ferramenta" para cumprir os objetivos do trabalho, indo de uso funcional ao disfuncional, no qual a própria substância passa a ser o problema. No Brasil, as evidências sobre a relação entre adição e uso de drogas no trabalho têm sido cada vez mais contundentes em contextos específicos de trabalho, tais como: operadores da bolsa de valores; motoristas de ônibus e caminhão; publicitários; jornalistas; médicos; enfermeiros; advogados e cortadores de cana (Lima, 2010)

Voltando-se a universitários trabalhadores, pode-se equiparar ao descrito por Fontaine (2006), como pessoas socialmente integradas e anônimas, que fazem o uso de estimulantes (anfetaminas, guaraná, café, tabaco), visando aumentar a capacidade de trabalho e concentração, alterar a noção de tempo, ainda que isto envolva sempre o risco. Ou também para afastar-se por meio do uso de SPAs, que modificam o estado de consciência (álcool e psicotrópicos ilícitos), que se intensifica nos últimos períodos letivos da faculdade ou quando o estresse do ambiente acadêmico se alia ao laboral (Lemos et al., 2007; Chiapetti \& Serbena, 2007). Estes usos foram iden- tificados por Lemos et al. (2007) e Pelicioli et al. (2017), em estudantes de medicina e da área de saúde que conjugam atividades teóricas e práticas.

Esse achado sobre o uso de café conduz a sugerir novos estudos com técnicas mais abertas que possam explorar a dinâmica do trabalho que o associa ao uso de tal substância. Que fatores culturais e de organização do trabalho estão implicados? Há fatores conjunturais como a estabilidade do emprego, as perspectivas de estabilidade e crescimento associados?

Por fim, todos esses resultados relativos à terceira questão podem ser vistos como desmitificando o papel do trabalho e da universidade no uso de SPAs, o que corrobora a afirmação de Fiorini et al. (2003) de que universidade não é o ponto de partida para início do uso de drogas. Entretanto, não podemos esquecer que a presente pesquisa apenas considerou se $\circ$ participante estava trabalhando ou não quando respondeu ao questionário. Entre os que trabalham há diversidades como, aquela derivada do tipo e qualidade do emprego, se emprego ou trabalho no setor informal, se trabalho em posição subordinada ou como empresário, se um microempresário ou o proprietário de um empreendimento maior, etc. Entre aqueles que não trabalham há também diversidades como, se nunca trabalhou, se está buscando trabalho/emprego, se perdeu um emprego recentemente e se não precisa de trabalhar enquanto estuda entre outras possibilidades. Sugerimos que essas diversidades de situações sejam consideradas em novas pesquisas.

\section{Considerações finais}

As SPAs utilizadas pelos universitários trabalhadores podem ser agrupadas e nomeadas em substâncias prescritas e não prescritas. Sendo que esses agrupamentos (fatores) não incluem o café, por suas características de aceitação social. Proporcionalidades diferentes para cada SPA foram verificadas, tanto para tipo e frequência de uso. Dentre as três variáveis verificadas na pesquisa (idade, sexo e situação de trabalho), apenas o sexo ofereceu predição para o uso de Substâncias Não Prescritas nos homens e Substâncias Prescritas para as mulheres. $O$ 
café apresentou interferência dos que trabalham e que possuem maior idade. Aponta-se uma desmitificação do papel do trabalho no uso de SPA.

Em relação as limitações, a escala de agrupamento das SPAs demanda adaptações para estudos futuros, no tocante da técnica de informação de uso com base em seus pares e não do indivíduo, a fim de diminuir a contaminação da resposta, assim como, a adequação dos níveis da escala de frequência de uso mais detalhada. Existe a demanda de considerar agrupamentos específicos de trabalhadores universitários e a sua relação com o uso de SPAs, que não foi possível devido a amostra heterogênea.

Esta pesquisa alcançou o objetivo de investigar o uso de substâncias psicoativas entre universitários trabalhadores de duas faculdades privadas de Belo Horizonte/MG analisando a relação entre a frequência do uso de substâncias e três variáveis antecedentes. Os resultados encontrados bem como as limitações poderão ser ponto de partida para a construção de um instrumento mais adequado para levantar o uso de SPAs em universitários, bem como para estimular novas reflexões sobre o impacto da condição de ser estudante universitário e trabalhador ao mesmo tempo.

\section{Conflitos de interesses}

Nenhum conflito financeiro, legal ou político envolvendo terceiros (governo, empresas e fundações privadas, etc.) foi declarado para nenhum aspecto do trabalho submetido (incluindo mas não limitandose a subvenções e financiamentos, conselho consultivo, desenho de estudo, preparação de manuscrito, análise estatística, etc).

\section{Referências}

Andrade, A. G., Duarte, P. C. A. V., \& Oliveira, L. G. (Orgs.). (2010). I Levantamento nacional sobre o uso de álcool, tabaco e outras drogas entre universitários das 27 capitais brasileiras. Brasília: SENAD. Recuperado de http://www.grea.org.br/userfiles/GREA-ILevantament oNacionalUniversitarios.pdf
Lei $n^{\circ} 12.852$, de 5/08/2013. Institui o estatuto da juventude e dispõe sobre os direitos dos jovens, os princípios e diretrizes das políticas públicas de juventude e o sistema nacional de juventude - Sinajuve. Recuperado de: http://www.planalto.gov.br/CCIVIL_03/_ Ato2011-2014/2013/Lei/I1 2852.htm

Chiapetti, N., \& Serbena, C. A. (2007). Uso de álcool, tabaco e drogas por estudantes da área de saúde de uma universidade de Curitiba. Psicologia: Reflexão e Crítica, 20(2), 303-313. Recuperado de http:// www.scielo.br/pdf/prc/v20n2/a17v20n2.pdf. doi: 10.1590/S0102-79722007000200017

Cogollo-Milanés, Z., Arrieta-Vergara, K. M., Blanco-Bayuelo, S., Ramos-Martínez, L., Zapata, K., \& Rodríguez-Berrio, Y. (2011). Public university students' psychosocial factors associated with legal and illegal substance consumption. Revista de Salud Pública, 13(3), 470-479. Recuperado de http://www.scielo.org.co/pdf/rsap/ v13n3/v13n3a09.pdf

Dázio, E. M. R., Zago, M. M. F., \& Fava, S. M. C. L. (2016). Use of alcohol and other drugs among male university students and its meanings. Revista da Escola de Enfermagem da USP, 50(5), 786-792. Recuperado de http://www.scielo.br/pdf/reeusp/v50n5/pt_00806234-reeusp-50-05-0786.pdf. doi: 10.1590/s0080623420160000600011

Díaz, E. S. M., \& Gómez, D. A. D. (2007). Una aproximación psicosocial al estrés escolar. Educación y Educadores, 10(2), 11-22. Recuperado de http:// educacionyeducadores.unisabana.edu.co/index.php/ eye/article/view/687/770

Eckschmidt, F., Andrade, A. G., \& Oliveira, L. G. (2013). Comparação do uso de drogas entre universitários brasileiros, norte-americanos e jovens da população geral brasileira. Jornal Brasileiro de Psiquiatria [online], 62(3), 199-207. Recuperado de http://www. scielo.br/pdf/ibpsiq/v62n3/04.pdf. doi: 10.1590/ S0047-20852013000300004

Edwards, G., Arif, A., \& Hadgson, R. (1981). Nomenclature and classification of drug and alcohol-related problems: A WHO memorandum. Bulletin of the World Health Organization. 59(2), 225-242. Recuperado de https://www.ncbi.nlm.nih.gov/pubmed/6972816

Fiorini, J. E., Alves, A. L., Ferreira, L. R., Fiorini, C. M., Durães, S. W., Santos, R. L. D., . . Ortiz, C. F. (2003). Use of licit and illicit drugs at the University of Alfenas. Revista do Hospital das Clínicas da Faculdade de Medicina da Universidade de São Paulo, 58(4), 199-206. Recuperado de http://www.scielo.br/ pdf/rhc/v58n4/17222.pdf. doi: 10.1590/S0041 $\underline{87812003000400003}$ 
Fonseca-Pedrero, E., Ortuño-Sierra, J., Paino, M., \& Muñiz, J. (2016). Experiencias psicóticas atenuadas y consumo de sustancias en universitarios. Adicciones, 28(3), 144 153. Recuperado de http://www.adicciones.es/index. php/adicciones/article/view/781

Fontaine, A. (2006). Double vie: Les drogues et le travail. Paris: Les empêcheurs de penser em rond.

Lemos, K. M., Neves, N. M. B. C., Kuwano, A. Y., Tedesqui, G., Bitencourt, A. G. V., Neves, F. B. C. S., .. . Lima, M. M. (2007). Uso de substâncias psicoativas entre estudantes de medicina de Salvador (BA). Revista de Psiquiatria Clínica, 34(3), $118-124$. Recuperado de http://www.scielo.br/pdf/rpc/v34n3/a03v34n3.pdf. doi: $10.1590 /$ S0101-60832007000300003

Lima, C. A. G., Maia, M. F. M., Magalhães, T. A., Oliveira, L. M. M., Reis, V. M. C. P., Brito, M. F. S. F., .. Silveira, L. P. M. F. (2017). Prevalência e fatores associados a comportamentos de risco à saúde em universitários no norte de Minas Gerais. Cadernos Saúde Coletiva, 25(2), 183-191. Recuperado de http://www.scielo. $\mathrm{br} / \mathrm{pdf} / \mathrm{cadsc} / \mathrm{v} 25 \mathrm{n} 2 / 1414-462 \mathrm{X}$-cadsc-1414462X201700020223.pdf. doi: 10.1590/1414$\underline{462 \times 201700020223}$

Lima, M. E. A. (2010). Dependência química e trabalho: Uso funcional e disfuncional de drogas nos contextos laborais. Revista Brasileira de Saúde Ocupacional, 35(1 22), 260-268. Recuperado de http://www. scielo.br/pdf/rbso/v35n 1 22/a08v35n 1 22.pdf. doi: $10.1590 /$ S0303-76572010000200008

Mardegan, P. S., Souza, R. S., Buaiz, V., \& Siqueira, M. M. (2007). Uso de substâncias psicoativas entre estudantes de enfermagem. Jornal Brasileiro de Psiquiatria [online], 56(4), 260-266. Recuperado de http://www.scielo.br/pdf/ibpsiq/v56n4/a04v56n4. pdf. doi: $10.1590 /$ S0047-20852007000400004

Martins, E. R. C., \& Zeitoune, R. C. G. (2007). As condições de trabalho como fator desencadeador do uso de substâncias psicoativas pelos trabalhadores de enfermagem. Escola Anna Nery [online], 11 (4), 639. 644. Recuperado de http://www.scielo.br/pdf/ ean/v1 ln4/v1 ln4a13.pdf. doi: 10.1590/S141481452007000400013

Nicastri, S. (2008). Drogas: Classificação e efeitos no organismo. In Prevenção ao uso indevido de drogas: Curso de capacitação para conselheiros municipais (pp. 20-29). Brasília: SENAD.
Oliveira, E. B., Cunningham, J., Strike, C., Brands, B., \& Wright, M. G. M. (2009). Normas percebidas por estudantes universitários sobre o uso de álcool pelos pares. Revista Latino-Americana de Enfermagem, 17/n. especial), 878-885. Recuperado de http://www.scielo. $\mathrm{br} / \mathrm{pdf} / \mathrm{rlae} / \mathrm{v17nspe/19.pdf.} \mathrm{doi:} \mathrm{10.1590/S0104-}$ 11692009000700019

Padovani, R. C., Neufeld, C. B., Maltoni, J., Barbosa, L. N. F., Souza, W. F., Cavalcanti, H. A. F., ... Lameu, J. N. (2014). Vulnerabilidade e bem-estar psicológicos do estudante universitário. Revista Brasileira de Terapias Cognitivas, 10(1), 2-10. Recuperado de http:// www.rbtc.org.br/detalhe_artigo.asp?id=188. doi: $10.5935 / 1808-5687.20140002$

Pelicioli, M., Barelli, C., Gonçalves, C. B. C., Hahn, S. R., \& Scherer, J. I. (2017). Perfil do consumo de álcool e prática do beber pesado episódico entre universitários brasileiros da área da saúde. Jornal Brasileiro de Psiquiatria, 66(3), 150-156. Recuperado de http:// www.scielo.br/pdf/ibpsiq/v66n3/0047-2085jbpsiq-66-3-0150.pdf. doi: 10.1590/0047$\underline{2085000000164}$

Pereira, D. S., Souza, R. S., Buaiz, V., \& Siqueira, M. M. (2008). Uso de substâncias psicoativas entre universitários de medicina da Universidade Federal do Espírito Santo. Jornal Brasileiro de Psiquiatria, 57(3), 188-195. Recuperado de http://www.scielo.br/ pdf/ibpsiq/v57n3/06.pdf . doi: 10.1590/S0047. $\underline{20852008000300006}$

Picolotto, E., Libardoni, L. F. C., Migott, A. M. B., \& Geib, L. T. C. (2010). Prevalência e fatores associados com o consumo de substâncias psicoativas por acadêmicos de enfermagem da Universidade de Passo Fundo. Ciência \& Saúde Coletiva, 15(3), 645-654. Recuperado de http://www.scielo.br/pdf/csc/v15n3/v15n3a06.pdf. doi: $10.1590 /$ S1413-81232010000300006

Silva, E. C., \& Tucci, A. M. (2015). Intervenção breve para redução do consumo de álcool e suas consequências em estudantes universitários brasileiros. Psicologia: Reflexão e Crítica, 28(4), 728-736. Recuperado de http://www.scielo.br/pdf/prc/v28n4/01027972-prc-28-04-00728.pdf. doi: 10.1590/16787153.201528410

Teixeira, R. S., Souza, R. S., Buaiz, V., \& Siqueira, M. M. (2010). Uso de substâncias psicoativas entre estudantes de odontologia da Universidade Federal do Espírito Santo. Ciência \& Saúde Coletiva, 15(3), 655-662. Recuperado de http://www.scielo.br/pdf/ csc/v15n3/v15n3a07.pdf. doi: 10.1590/s141381232010000300007

Workforall Consortium (2014). T-PSlab Survey. Salud y bienestar en trabajadores y desempleados. Un estudio internacional. Oviedo. 